Feature selection for classification of hyperspectral data by SVM

Pal, M. and Foody, G. M.

IEEE Transactions on Geoscience and Remote Sensing, 48, 2297-2307 (2010)

The manuscript of the above article revised after peer review and submitted to the journal for publication, follows. Please note that small changes may have been made after submission and the definitive version is that subsequently published as:

Pal, M. and Foody, G. M., 2010. Feature selection for classification of hyperspectral data by SVM, IEEE Transactions on Geoscience and Remote Sensing, 48, 2297-2307. 


\title{
Feature selection for classification of hyperspectral data by SVM
}

\author{
Mahesh Pal ${ }^{11}$ and Giles M. Foody ${ }^{2}$, Member IEEE \\ ${ }^{1}$ Department of Civil Engineering, NIT Kurukshetra, Haryana, 136119, INDIA \\ ${ }^{2}$ School of Geography, University of Nottingham, Nottingham, NG7 2RD, UK
}

\begin{abstract}
SVM are attractive for the classification of remotely sensed data with some claims that the method is insensitive to the dimensionality of the data and so not requiring a dimensionality reduction analysis in pre-processing. Here, a series of classification analyses with two hyperspectral sensor data sets reveal that the accuracy of a classification by a SVM does vary as a function of the number of features used. Critically, it is shown that the accuracy of a classification may decline significantly (at 0.05 level of statistical significance) with the addition of features, especially if a small training sample is used. This highlights a dependency of the accuracy of classification by a SVM on the dimensionality of the data and so the potential value of undertaking a feature selection analysis prior to classification. Additionally, it is demonstrated that even when a large training sample is available feature selection may still be useful. For example, the accuracy derived from the use of a small number of features may be noninferior (at $0.05 \%$ level of significance) to that derived from the use of a larger feature set providing potential advantages in relation to issues such as data storage and computational processing costs. Feature selection may, therefore, be a valuable analysis to include in pre-processing operations for classification by a SVM.
\end{abstract}

Keywords: feature selection, support vector machines, Hughes phenomenon, hyperspectral data, classification accuracy

\footnotetext{
${ }^{1}$ Corresponding author, e-mail: mpce_pal@yahoo.co.uk, fax: +91 1744238050
} 


\section{INTRODUCTION}

Progress in hyperspectral sensor technology allows the measurement of radiation in the visible to the infrared spectral region in many finely spaced spectral features or wavebands. Images acquired by these hyperspectral sensors provide greater detail on the spectral variation of targets than conventional multispectral systems, providing the potential to derive more information about different objects in the area imaged [1]. Analysis and interpretation of data from these sensors presents new possibilities for applications such as land cover classification [2]. However, the availability of large amounts of data also represents a challenge to classification analyses. For example, the use of many features may require the estimation of a considerable number of parameters during the classification process [3]. Ideally, each feature (e.g. spectral waveband) used in the classification process should add an independent set of information. Often, however, features are highly correlated and this can suggest a degree of redundancy in the available information which may have a negative impact on classification accuracy [4].

One problem often noted in the classification of hyperspectral data is the Hughes effect or phenomenon. The latter can have a major negative impact on the accuracy of a classification. The key characteristics of the phenomenon, assuming a fixed training set, may be illustrated for a typical scenario in which features are incrementally added to a classification analysis. Initially, classification accuracy increases with the addition of new features. The rate of increase in accuracy, however, declines and eventually accuracy will begin to decrease as more features are included. Although it may at first seem counter-intuitive for the provision of additional discriminatory information to result in a loss of accuracy the problem is often encountered [5-7] and arises as a consequence of the analysis requiring the estimation of more parameters from the 
(fixed) training sample. Thus the addition of features may lead to a reduction in classification accuracy [8].

The Hughes phenomenon has been observed in many remote sensing studies based upon a range of classifiers $[3,5,9,10]$. For example, a parametric technique, such as the maximum likelihood classifier, may not be able to classify a data set accurately if the ratio of sample size to number of features is small as it will not be able to correctly estimate the first and second order statistics (i.e. mean and covariance) that is fundamental to the analysis [6]. Note that with a fixed training set size, this ratio declines as the number of features is increased. Thus, two key attributes of the training set are its size and fixed nature. If, for example, the training set was not fixed but was instead increased appropriately with the addition of new features, the phenomenon may not occur. Similarly, if the fixed training set size was very large, so that even when all features of a hyperspectral sensor were used, the Hughes effect may not be observed as all parameters may be estimated adequately. Unfortunately, however, the size of the training set required for accurate parameter estimation may exceed that available to the analyst. Given that training data acquisition may be difficult and costly [11-13] some means to accommodate the negative issues associated with high dimensional data sets is required.

Various approaches could be adopted for the appropriate classification of high dimensional data. These span a spectrum from the adoption of a classifier that is relatively insensitive to the Hughes effect [14] through the use of methods to effectively increase training set size $[5,11]$ to the application of some form of dimensionality reduction procedure prior to the classification analysis. It may also sometimes be appropriate to use a combination of approaches to reduce the possibility of the Hughes effect being observed. The precise approach adopted may vary with study objectives, data sets and classification approach. One classification method that has been claimed to 
be independent of the Hughes effect and so promoted for use with hyperspectral data sets is the support vector machine (SVM; [15]) although, as will be discussed below, there is some uncertainty relating to the role of feature reduction with this method.

The SVM has become a popular method for image classification. It is based on structural risk minimisation and exploits a margin-based criterion that is attractive for many classification applications [16]. In comparison to approaches based on empirical risk, which minimise the misclassification error on the training set, structural risk minimisation seeks the smallest probability of misclassifying a previously unseen data point drawn randomly from a fixed but unknown probability distribution. Furthermore, a SVM tries to find an optimal hyperplane that maximises the margin between classes by using a small number of training cases, the support vectors. The complexity of SVM depends only on these support vectors and it is argued that the dimensionality of the input space has no importance $[15,17,18]$. This hypothesis has been supported by a range of studies with SVM such as those employing the popular radial basis function kernel for land cover classification applications [19, 20, 21].

The basis of the SVM and results of some studies, therefore, suggest that SVM classification may be unaffected by the dimensionality of the data set and so number of features used. However, other studies have shown that the accuracy of SVM classification could still be increased by reducing the dimensionality of the data set [22, 23], hence there is a degree of uncertainty over the role of feature reduction in SVM based classification. Feature reduction, however, impacts on more than just the accuracy of a classification. A feature reduction analysis may be undertaken for a variety of reasons. For example, it may speed-up the classification process by reducing data set size and may increase the predictive accuracy as well as ability to understand the classification rules [24]. It may also simply provide advantages in terms of reducing 
data storage requirements. Feature reduction may, therefore, still be a useful analysis even if it has no positive effect on classification accuracy.

Two broad categories of feature reduction techniques are commonly encountered in remote sensing: feature extraction and feature selection [25, 26]. With feature extraction, the original remotely sensed data set is typically transformed in some way that allows the definition of a small set of new features which contain the vast majority of the original data set's information. More popular, and the focus of this paper, are feature selection methods. The latter aim to define a sub-set of the original features which allows the classes to be discriminated accurately. That is, feature selection typically aims to identify a subset of the original features that maintains the useful information to separate the classes with highly correlated and redundant features excluded from the classification analysis [25].

Feature selection procedures are dependent on the properties of the input data as well as on the classifier used [27, 28]. These procedures require a criterion be defined by which it is possible to judge the quality of each feature in terms of its discriminating power [29]. A computational procedure is then required to search through the range of potential subsets of features and select the 'best' subset of features based upon some pre-defined criterion. The search procedure could simply consist of an exhaustive search over all possible subsets of features since this is guaranteed to find the optimal subset. In a practical application, however, the computational requirements of this approach are unreasonably large and a non-exhaustive search procedure is usually used [30]. A wide variety of feature selection methods have been applied to remotely sensed data [30-33]. Based on whether or not they use classification algorithms to evaluate subsets, the different methods can be grouped into three categories: filters, wrappers and embedded approaches. These approaches may select different subsets and these in turn may vary in suitability for use as a pre-processing algorithm for different classifiers. Because of 
these differences and the range of reasons for undertaking a feature selection as well as the numerous issues that influence outputs and impact on later analyses feature selection remains a topic for research [34].

Although the literature includes claims that classification by SVM is insensitive to the Hughes effect $[19-21,35]$ it also includes case studies using simulated data [36, 37] and theoretical arguments that indicate a positive role for feature selection in SVM classification [38, 39]. Both [38] and [39] based their arguments on the use of local kernels, such as the popular radial basis function, with kernel based classifiers in which the cases lying in the neighbourhood of the case being used to calculate the kernel value have a large influence [40]. In their argument, [38] used the bias-variance dilemma [41] to suggest that the classifiers with local kernel would require exponentially large training data set to have same level of classification error in high dimensional space as that in a lower space, suggesting the sensitivity of SVM classifier to the curse of dimensionality. On the other hand, [39] suggested that locality of a kernel is an important property that makes the generated model more interpretable and used algorithm more stable than the algorithms using global kernels. They argued that a radial basis function kernel loses the properties of a local kernel with increasing feature space, a reason why they may be unsuitable in high dimensional space. With the latter, for example, it has been argued that classifiers using local kernels are sensitive to the curse of dimensionality as the properties of learned function at a case depends on its neighbours, which fails to work in high dimensional space. There is, therefore, uncertainty in the literature over the sensitivity of classification by a SVM to the dimensionality of the data set and so of the value of feature selection within such an analysis.

This paper aims to address key aspects of this uncertainty associated with the role of feature selection in the classification of hyperspectral data sets. Specifically, the 
paper aims to explore the relationship between the accuracy of classification by a SVM and the dimensionality of the input data. The later will also be controlled through application of a series of feature selection methods and so also highlight the impact, if any, of different feature selection techniques on the accuracy of SVM-based classification. Variation in the accuracy of classifications derived using feature sets of differing size will be evaluated using statistical tests of difference and non-inferiority $[42,43]$ in order to evaluate the potential role of feature selection in SVM-based classification. This paper is, to our knowledge, the first rigorous assessment of the Hughes effect on SVM with hyperspectral dataset. Other studies [e.g. 19 20, 21] have commented on the Hughes effect in relation to SVM-based classification of remotely sensed data but this paper differs in that the experimental design adopted gives an opportunity for the effect to occur (e.g. by including analyses based on small training sets) and the statistical significance of differences in accuracy is evaluated rigorously (e.g. including formal tests for the difference and non-inferiority of accuracy). To set the context to this work, section II briefly outlines classification by a SVM. Section III provides a summary of the main methods and data sets used. Section IV presents the results and section $\mathrm{V}$ details the conclusions of the research undertaken.

\section{SVM}

The SVM is based on statistical learning theory [14] and seeks to find an optimal hyperplane as a decision function in high dimensional space $[44,45]$. In the case of a two-class pattern recognition problem in which the classes are linearly separable, the SVM selects from among the infinite number of linear decision boundaries the one that minimises the generalisation error. Thus, the selected decision boundary (represented by a hyperplane in feature space) will be one that leaves the greatest margin between the two classes, where margin is defined as the sum of the 
distances to the hyperplane from the closest cases of the two classes [14]. The problem of maximising the margin can be solved using standard quadratic programming optimisation techniques.

The simplest scenario for classification by a SVM is when the classes are linearly separable. This scenario may be illustrated with the training data set comprising $k$ cases be represented by $\left\{\mathbf{x}_{\mathrm{i}}, y_{i}\right\}, \mathrm{i}=1, \ldots, k$, where $x \in \mathbf{R}^{\mathrm{N}}$ is an $N$-dimensional space and $y \in\{-1,+1\}$ is the class label. These training patterns are linearly separable if there exists a vector $\mathbf{w}$ (determining the orientation of a discriminating plane) and a scalar $b$ (determining the offset of the discriminating plane from the origin) such that

$$
y_{i}\left(\mathrm{w} \cdot \mathrm{x}_{i}+b\right)-1 \geq 0
$$

The hypothesis space can be defined by the set of functions given by

$$
f_{\mathbf{w}, b}=\operatorname{sign}(\mathbf{w} \cdot \mathbf{x}+b)
$$

The SVM finds the separating hyperplanes for which the distance between the classes, measured along a line perpendicular to the hyperplane, is maximised. This can be achieved by solving following constrained optimization problem

$$
\min _{w, b} \frac{1}{2}\|\mathbf{w}\|^{2}
$$

For linearly non-separable classes, the restriction that all training cases of a given class lie on the same side of the optimal hyperplane can be relaxed by the introduction of a 'slack variable' $\xi_{\mathrm{i}} \geq 0$. In this case, the SVM searches for the hyperplane that maximises the margin and that, at the same time, minimises a quantity proportional to the number of misclassification errors. This trade-off between margin and misclassification error is controlled by a positive constant $C$ such that $\infty>C>0$. Thus, for non-separable data, (3) can be written as: 


$$
\min _{\mathrm{w}, b, \xi_{1}, \ldots . \xi_{k}}\left[\frac{1}{2}\|\mathbf{w}\|^{2}+C \sum_{i=1}^{k} \xi_{i}\right]
$$

For non-linear decision surfaces, a feature vector, $\mathrm{x} \in \mathbf{R}^{N}$ is mapped into a higher dimensional Euclidean space (feature space) $F$, via a non-linear vector function $\Phi: \mathbf{R}^{N} \mapsto F[44]$. The optimal margin problem in $F$ can be written by replacing $\mathbf{x}_{i} \cdot \mathbf{x}_{j}$ with $\boldsymbol{\Phi}\left(\mathbf{x}_{i}\right) \cdot \boldsymbol{\Phi}\left(\mathbf{x}_{j}\right)$ which is computationally expensive. To address this with problem, [14] introduced the concept of using a kernel function $K$ in the design of non-linear SVMs. A kernel function is defined as:

$$
K\left(\mathbf{x}_{i}, \mathbf{x}_{j}\right)=\boldsymbol{\Phi}\left(\mathbf{x}_{i}\right) \cdot \boldsymbol{\Phi}\left(\mathbf{x}_{j}\right)
$$

and with the use of a kernel function equation (2) becomes:

$$
f(\mathrm{x})=\operatorname{sign}\left(\sum_{i} \lambda_{i} y_{i} \mathrm{~K}\left(\mathbf{x}_{\mathrm{i}}, \mathbf{x}_{j}\right)+b\right)
$$

where $\lambda_{i}$ is a Lagrange multiplier. A detailed discussion of the computational aspects of SVM can be found in $[14,45]$ with many examples also in the remote sensing literature $[19,21,46,47]$.

\section{DATA AND METHODS}

\section{A. Test Areas}

Data sets for two study areas were used. The first study area, La Mancha Alta, lies to the south of Madrid, Spain. It is an area of Mediterranean semi-arid wetland, which supports rain-fed cultivation of crops such as wheat, barley, vines and olives. A hyperspectral image data set was acquired for the test site by the DAIS 7915 sensor on 29 June 2000. The sensor was a 79- channel imaging spectrometer developed and operated by the German Space Agency [48]. This instrument operated at a spatial resolution of $5 \mathrm{~m}$ and acquired data in the wavelength range $0.502-12.278 \mu \mathrm{m}$. Attention here focused on the data acquired in only the visible and near-infrared spectrum. Thus 
the data acquired in the 7 features located in the mid- and thermal infrared region were removed. Of the remaining 72 features covering spectral region $0.502-2.395 \mu \mathrm{m}$ a further 7 features were removed because of striping noise distortions in the data. The features removed were bands $41(1.948 \mu \mathrm{m}), 42(1.964 \mu \mathrm{m})$ and 68-72 $(2.343-2.395$ $\mu \mathrm{m})$. After these pre-processing operations, an area of 512 pixels by 512 pixels from the remaining 65 features covering the test site was extracted for further analysis.

The second study area was a region of agricultural land in Indiana, USA. For this site a hyperspectral dataset acquired by Airborne Visible/Infrared Imaging Spectrometer (AVIRIS) was used. This data set is available online from [49]. The data set consists of a scene of size 145 pixels x 145 columns. Of the 220 spectral bands acquired by the AVIRIS sensor, 35 were removed as they were affected by noise. For ease of presentation, the bands used were re-numbered 1-65 and 1-185 in order by increasing wavelength for the DAIS and AVIRIS data sets respectively.

\section{B. Training and Testing Data Sets}

For the DAIS data set, field observations of the test site were undertaken in late June 2001, exactly 1 year after the image data were acquired, to generate a ground reference data set. Visual examination of DAIS imagery combined with field experience showed that the region comprised mainly eight land cover types: wheat, water, salt lake, hydrophytic vegetation, vineyards, bare soil, pasture and built-up land. A ground reference image was generated from the field information. With the AVIRIS data set, a ground reference image available on [49] was used to collect the training and test pixels for a total of nine land cover classes (corn-no till, corn-min till, grass/pasture, grass/trees, hay-windrowed, soybeans-no tills, soybeans-min till, soybean-clean and woods). Stratified random sampling, by class, was undertaken in order to collect 
independent data sets for training (up to 100 pixels per-class) and testing the SVM classifications of the DAIS and AVIRIS data sets.

To evaluate the sensitivity of the SVM to the Hughes effect, a series of training sets of differing sample size were acquired. These data sets were formed by selecting cases randomly from the total available for training each class. A total of six training set sizes, comprising $8,15,25,50,75$ and 100 pixels per-class, was used. These training samples are typical of the sizes used in remote sensing studies [e.g., 26, 46, 50, 51, 52, 53] but critically also include small sizes at which the Hughes effect would be expected to manifest itself, if at all. For each size of training set, except that using all 100 pixels available for each class, five independent samples were derived from the available training data. Each of the five training sets of a given size was used to train a classification and, to avoid extreme results, the main focus here is on the classification with the median accuracy.

SVM classifications using training sets of differing size were undertaken in which the dimensionality of the input data set, indicated by the number of features used, was varied. Since the main concern was to determine if the Hughes effect would be observed and not the design of an optimal classification, most attention focused on the scenario in which the features were entered in a single fashion for comparative purposes. With this, features were added incrementally in groups of 5 in order of wavelength. Thus, the first analysis used features 1-5, the second features 1-10 and so on until all the thirteenth and thirty seven analyses with DAIS and AVIRIS data respectively. A number of additional analyses were undertaken with DAIS data in which features were added individually in order of decreasing discriminatory power (i.e. the feature estimated to provide most discriminatory information was entered first and that which provided the least discriminatory information was added last). Irrespective of 
the method of incrementing features, the accuracy with which an independent testing set was classified was calculated at each incremental step.

Classification accuracy was estimated using a testing set that comprised a sample of 3800 pixels (500 pixels for seven classes and 300 pixels for the relatively scarce pasture class) with the DAIS data and 3150 pixels (350 pixels per class) with the AVIRIS data sets. In all cases, accuracy was expressed as the percentage of correctly allocated cases. The statistical significance of differences in accuracy was assessed using the McNemar test and confidence intervals [43, 54, 55]. Two types of test were undertaken to elucidate the effect of feature selection on SVM classification accuracy. First, the statistical significance of differences in accuracy was evaluated. This testing was undertaken because one characteristic feature of an analysis that is sensitive to the Hughes effect is a decrease in accuracy following the inclusion of additional features. Thus, the detection of a statistically significant decrease in classification accuracy following the addition of features to the analysis would be indication of sensitivity to the Hughes effect. A standard one-sided (as the focus is on a directional alternative hypothesis) test of the difference in accuracy values was derived using the McNemar test [55]. However, as feature selection has positive impacts beyond those associated with classification accuracy (e.g. reduced data processing time and storage requirements) a positive role would also occur if a small feature set could be used without any significant loss of classification accuracy. This cannot be assessed with a test for difference, as a result indicating no significant difference in accuracy is not actually proof of similarity [56]. Indeed, in this situation the desire is not to test for a significant difference in accuracy but rather to test for the similarity in accuracy, which could be met in this situation through the application of a test for non-inferiority [42, 43]. In essence, the aim is to determine if a small feature set, which provides advantages to the analyst, can be used to derive a classification as accurate as that from a large or 
indeed the full feature set. The latter test for non-inferiority was achieved using the confidence interval fitted to the estimated differences in classification accuracy [43]. For the purpose of this paper it was assumed that a $1.00 \%$ decline in accuracy from the peak value was of no practical significance and this value taken to define the extent of the zone of indifference in the test. Critically, a positive role for feature selection analyses would be indicated if the test for difference was significant (showing that accuracy can be degraded by the addition of new features) and/or if the test for noninferiority was significant (showing that a small feature set derives a classification as accurate as that from the use of a large feature set but providing advantages in relation to data storage and processing etc.).

\section{Feature Selection Algorithms}

From the range of feature selection methods available, four established methods, including one from each of the main categories of method identified above, were applied to the DAIS data. The salient issues of each method is briefly outlined below.

\section{1) $S V M-R F E$}

The Support Vector Machine Recursive Feature Elimination (SVM-RFE) is a wrapper based approach utilising the SVM as base classifier [22]. The SVM-RFE utilise the objective function $(1 / 2)\|\mathbf{w}\|^{2}$ as a feature ranking criterion to produce a list of features ordered by apparent discriminatory ability. At each step, the coefficients of the weight vector $\mathbf{w}$ are used to compute the ranking scores of all features remaining. The feature, with the smallest ranking score $\left(w_{i}\right)^{2}$ is eliminated, where $w_{i}$ represents the corresponding $i$-th component of $\mathbf{w}$. This approach to feature selection, therefore, uses a backward feature elimination scheme to recursively remove insignificant features (i.e. at 
each step the feature whose removal changes the objective function least is excluded) from subsets of features in order to derive a list of all features in rank order of value.

\section{2) Correlation-based Feature Selection}

Correlation-based feature selection (CFS) is a filter algorithm that selects a feature subset on the basis of a correlation-based heuristic evaluation function [57]. The heuristics by which CFS measures the quality of a set of features takes into account the usefulness of individual features for predicting the class and can be summarised as:

$$
\frac{f C_{c i}}{\sqrt{f+f(f-1) C_{i i}}}
$$

where $f$ is the number of features in the subset, $C_{c i}$ is the mean feature correlation with the class and $C_{i i}$ is the average feature inter-correlation. Both $C_{c i}$ and $C_{i i}$ are calculated by using a measure based on conditional entropy [58]. The numerator provides an indication of how predictive of the class a group of features are where as denominator indicates about the redundancy among the features. The evaluation criterion used in this algorithm is biased towards the feature subsets highly predictive of the class and not predictive of each other. This criteria acts to filter out the irrelevant features as they have low correlations with the class and redundant features are ignored as they will be highly correlated with one or more feature, thus providing a subset of best selected features. In order to reduce the computation cost, a bidirectional search (a parallel implementation of sequential forward and backward selection) may be used. This approach searches the space of feature subsets by greedy hill climbing in a way that features already selected by sequential forward selection are not removed by backward selection and the features already removed by backward selection are not selected by forward selection. 


\section{3) Minimum-Redundancy-Maximum-Relevance}

Minimum-Redundancy-Maximum-Relevance (mRMR) feature selection is a filter based method that uses mutual information to determine the dependence between the features [59]. The mRMR use a criterion which select features that are different from each other and still have largest dependency on the target class. This approach consists in selecting a feature $f_{i}$ among the not selected features $f_{S}$ that maximises $\left(u_{i}-r_{i}\right)$, where $u_{i}$ is the relevance of $f_{i}$ to the class $c$ alone and $r_{i}$ is the mean redundancy of $f_{i}$ to each of the already selected features. In term of mutual information, $u_{i}$ and $r_{i}$ can be defined as:

$$
\begin{aligned}
& u_{i}=\frac{1}{|f|} \sum_{f_{i} \in f} I\left(f_{i} ; c\right) \\
& r_{i}=\frac{1}{|f|^{2}} \sum_{f_{j} \in f} I\left(f_{i}, f_{j}\right)
\end{aligned}
$$

where $I(f ; c)$ is the mutual information between two random variables $f$ and $c$. At each step, this method selects a feature that has best compromised relevanceredundancy and can be used to produce a ranked list of all features in terms of discriminating ability.

\section{4) Random Forest}

The random forest based approach is an embedded method of feature selection. The random forest consists of a collection of decision tree classifiers [60], where each tree in the forest has been trained using a bootstrap sample of training data and a random subset of features sampled independently from the input features. A sub-set of the training data set is omitted from the training of each classifier [61]. These left out data are called out-of-bag (out of the bootstrap) samples and used for feature selection by determining the importance of different features during classification process $[60,62]$. The latter is based on a $Z$ score, which can be used to assign a significance level 
(importance level) to a feature and from this a ranked list of all features may be derived [60].

\section{Methods}

SVM were initially designed for binary classification problems. A range of methods have been suggested for multi-class classification [21, 63, 64]. One of these, the 'one against one' approach, was used here [65] with both hyperspectral datasets. Throughout, a radial basis function kernel was used with $\gamma$ (kernel width parameter) $=2$ and $C=5000$, values which were used successfully with the DAIS hyperspectral dataset in other studies $[19,20,33,66]$. For analyses of the AVIRIS dataset, a RBF kernel with $\gamma=1$ and regularisation parameter $C=50$ was used [66].

With the feature selection by random forests, one third of the total data set available for training was used to form the out-of-bag sample. The random forest classifier also requires finding optimal value of number of features used to generate a tree as well the total numbers of trees. After several trials, 13 features and 100 trees were found to be working well with the DAIS dataset [33].

\section{RESULTS}

The accuracy of classification by a SVM varied as a function of the number of features used and the size of the training set using DAIS dataset (Fig. 1). In general terms, classification accuracy tended to increase with an increase in the number of features. Critically, however, when a fixed training set of small size ( $\leq 25$ cases perclass) was used accuracy initially rose with the addition of features to a peak but thereafter declined with the addition of further features. Moreover, the decline in accuracy was statistically significant, even for the classification based on the largest

training set size (Table I). For example, the largest difference between the peak 
accuracy and that obtained from the use of all 65 features was $5.00 \%$, a difference that was significant at the 0.05 level of significance (Table I).

Similar general trends to those found with the analysis of the DIAS data were observed with the results of the analyses of the AVIRIS data set (Fig. 2). Critically, classification accuracy was observed to decline with the addition of features. Moreover, with this data set, a statistically significant (at 0.05 level) decline in accuracy with the addition of features was observed for all training set sizes (Table II). The largest difference between the peak accuracy and that obtained from the use of all 185 features was $8.36 \%$.

Consequently, the key negative characteristic of the curse of dimensionality or Hughes effect was observed with SVM classification when a small training set was used. Although this result contradicts some statements in the literature that suggest the SVM is independent of the dimensionality of the data set $[20,21]$ it should be noted that these studies used relatively large training sets and do not include a rigorous statistical test of the significance of differences in accuracy. For example, [21] used over 230 training cases for each class while [20] used sample sizes of at least 100 pixels perclass. The size of the training sets used in these studies may have been sufficiently large to ensure that Hughes effect was not manifest in the analyses reported. Thus, in these studies the experimental designs adopted may not have provided an opportunity for the Hughes effect to arise and be detected. Additionally, it may be expected that the degree to which the effect is observed may vary from study to study as a function of the classes (e.g. their number and spectral separability) and data set (e.g. number and location of spectral wavebands). Note, for example, that the Hughes effect appeared to occur at each training set size studied with the AVIRIS data (Fig. 2) but only when small $(\leq 25$ cases per-class) training sets were used with the DIAS data set (Fig. 1).

Insert Tables I-II here 
Insert Fig. 1- 2 here

Having established that the accuracy of classification by a SVM is sensitive to the number of features used, the four different feature selection methods were applied to the DIAS data in order to evaluate the sensitivity of SVM classification to different types of feature selection method. The aim was not to define an optimal feature selection but to provide insight into the sensitivity of the SVM classification to the method used.

The classifications derived after application of the four feature selection methods varied in accuracy. Unlike the previous analyses, features were added individually to classifications in the order suggested by the feature selection analysis. To focus on key trends, Table III shows the accuracy derived without feature selection and the accuracy that was of closest magnitude after the application of each of the feature selection methods. Critically, the table also identifies the number of features used to derive the classification accuracy closest to that derived when no feature selection was undertaken. Irrespective of feature selection algorithm employed, the results suggest that a small subset of selected features $(\leq 12)$ would be sufficient to achieve comparable accuracy with the small training sets comprising 8,15 and 25 pixels per-class. In comparison, the training sets with 50,75 and 100 pixels per-class requires a larger subset of selected features to achieve the comparable classification accuracy to that derived from the full dataset (and the accuracy values were also of a higher magnitude).

It was evident from Table III that the feature selection methods varied in the efficiency, measured in terms of the number of features required to derive a classification of comparable accuracy to that derived without feature selection. Note for example, that the two filter based feature selection approaches, the CFS uses a smaller subset of features in comparison to mRMR. This suggests, for this dataset at least, CFS is more suitable than the mRMR method. 


\section{Insert Table III here}

It was also evident that the specific features selected by the different methods varied. Table IV identifies the selected features that provided the classification of comparable accuracy to that derived from the full (65 features) dataset. It was evident that a dissimilar feature list was obtained from analyses based on training sets of differing size, with at most only three common features observed with any one feature selection method. The outputs of the feature selection methods was, therefore, a function of the training set size. Moreover, the lack of commonalities in features selected with different training set sizes also confirms that the best set of features selected by a nonexhaustive search need not to contain the best feature or a set of best features from the full feature space [67].

\section{Insert Table IV here}

For comparison against the results given in Fig. 1, Fig. 3-5 show the relationship between classification accuracy and number of selected features using three of the feature selection methods. The CFS based feature selection method was excluded from this analysis as this approach does not provide a ranked list of the features. For purpose of comparability with Fig. 1 the features have been added in groups of 5 (in order of discriminating ability). The statistical significance of the difference in accuracy between the peak accuracy value and that derived with the use of the full feature set for each classification summarised in Fig. 3-5 was evaluated with a McNemar test. The derived $Z$-values are provided in Table $\mathrm{V}$ which suggests a similar trend as achieved with earlier combination of features (Fig. 1) using the training sample size of 8, 15, and 25 pixels per class. It was evident, however, that the peak accuracy was derived with a smaller number of features as in this case features were added in order of discriminating power.

Insert Tables V-VIII here

Insert Fig. 3-5 here 
The results highlight that a statistically significant negative impact of feature set size on classification accuracy was observed when a small training sample was used; confirming the results of the McNemar test for a significant difference. Although this in itself points to a dependency of SVM classification on the dimensionality of the data set and highlights a positive role for feature selection analysis the latter has other advantages and the results suggest feature selection may be valuable even when a large training sample was available. Note, for example, that in all series of analyses (Fig. 1 and Fig. 3-5) when the largest training sample was used (100 cases per-class) the accuracy was largely maintained when the number of features is reduced from the full (65 features) to small sub-set; only at a very small number of features did classification accuracy decline markedly. This similarity in accuracy values shows that the positive benefits of feature selection (e.g. reduced data storage and processing requirements) may be achieved without significant negative effect on classification accuracy. The latter is evident in the results of the non-inferiority testing summarised in Tables VIVIII. Critically, the accuracy of classifications derived with the use of relatively small training sets was not statistically inferior to the peak accuracy derived from the use of a larger feature set size.

\section{CONCLUSIONS}

The SVM has been widely used and promoted for land cover classification studies including multispectral and hyperspectral data with some studies suggesting that the method is not affected by the Hughes phenomena. A major conclusion of this study is that the accuracy of SVM classification is influenced by the number of features used and so is affected by Hughes phenomenon with the impact most evident when a small training set is used (Fig. 1 and 2, Tables I and II). It is possible that the Hughes effect had not been observed in some other studies because the opportunity for it to become 
manifested in the results was limited through experimental design, notably through the use of a large training set. The results presented in this paper show that the accuracy of classification by a SVM can be significantly reduced by the addition of features and that the effect is most apparent with small training sets. With the AVIRIS data set, a significant reduction in accuracy with the addition of features was observed at all training set sizes evaluated. With the DIAS data set, a statistically significant decline in accuracy was also observed for small training sets ( $\leq 25$ cases per-class). However, even with a large training sample using DAIS dataset, feature selection may have a positive role, providing a reduced data set that may be used to yield a classification of similar accuracy to that derived from use of a much larger feature set. As the accuracy of SVM classification was dependent on the dimensionality of the data set and the size of the training set it may, therefore, be beneficial to undertake a feature selection analysis prior to a classification analysis. The results, however, also highlight that the choice of feature selection methods may be important. For example, the results derived from analyses with four different feature selection methods show that the number of features selected varied greatly.

\section{Acknowledgements}

The DAIS data were collected and processed by DLR and were kindly made available by Prof. J. Gumuzzio of the Autonomous University of Madrid, Spain. Mahesh Pal thanks the Association of Commonwealth Universities (ACU), London, for providing a fellowship to fund this research and the School of Geography, University of Nottingham for computing facilities. We are also grateful to the three referees for their constructive comments on the original version of this paper. 


\section{REFERENCES}

[1] C.-I Chang, Hyperspectral Data Exploitation: Theory and Applications. New Jersey: John Wiley and Sons, 2007.

[2] J. B. Campbell, Introduction to Remote Sensing. Third edition, New York: The Guilford press, 2002.

[3] J. A. Benediktsson and J. R. Sveinsson, "Feature extraction for multisource data classification with artificial, neural networks," International Journal of Remote Sensing, vol. 18, no. 4, pp. 727-740, March 1997.

[4] P. Zhong, P. Zhang, and R. Wang, "Dynamic learning of SMLR for feature selection and classification of hyperspectral data," IEEE Geoscience and Remote Sensing Letters, vol. 5, no. 2, pp. 280-284, April 2008.

[5] B. M. Shahshahani and D. A. Landgrebe, "The effect of unlabeled samples in reducing the small sample size problem and mitigating the Hughes phenomenon," IEEE Transactions on Geoscience and Remote Sensing, vol. 32, no. 5, pp.10871095, Sept.1994.

[6] S. Tadjudin and D.A. Landgrebe, "Covariance estimation with limited training samples," IEEE Transactions on Geoscience and Remote Sensing, Vol. 37, no. 4, pp. 2113-2118, July 1999.

[7] M. Chi, R. Feng, and L.Bruzzone, "Classification of hyperspectral remote-sensing data with primal SVM for small-sized training dataset problem," Advances in Space Research, vol. 41, no. 4, pp. 1793-1799, 2008.

[8] G. F. Hughes, "On the mean accuracy of statistical pattern recognizers," IEEE Transactions on Information Theory, IT-14, pp. 55-63, 1968. 
[9] S. Lu, K. Oki, Y. Shimizu, and K. Omasa, "Comparison between several feature extraction/classification methods for mapping complicated agricultural land use patches using airborne hyperspectral data," International Journal of Remote Sensing, vol. 28, no. 5, pp. 963-984, Jan. 2007.

[10] S. Tadjudin and D. A. Landgrebe, "A decision tree classifier design for highdimensional data with limited training samples," IEEE Geoscience and Remote Sensing Symposium, Vol. 1, pp. 790-792, 27-31 May 1996.

[11] M. Chi and L. Bruzzone, “A semilabeled-sample-driven bagging technique for illposed classification problems," IEEE Geosciences Remote Sensing Letters, vol. 2, no. 1, pp. 69-73, January 2005.

[12] P. Mantero, G. Moser and S.B. Serpico, "Partially supervised classification of remote sensing images through SVM-based probability density estimation," IEEE Transactions on Geoscience and Remote Sensing, vol. 43, no. 3, pp. 559-570, March 2005.

[13] G. M. Foody and A. Mathur, "Toward intelligent training of supervised image classifications: directing training data acquisition for SVM classification," Remote Sensing of Environment, vol. 93, no. 1-2, pp. 107-117, Oct. 2004.

[14] V. N. Vapnik, The Nature of Statistical Learning Theory. New York: SpringerVerlag, 1995.

[15] C. Cortes and V. N. Vapnik, "Support vector networks," Machine Learning, vol. 20, no. 3, pp. 273-297, Sept. 1995.

[16] V. N. Vapnik, Estimation of Dependences Based on Empirical Data. New York: Springer Verlag, 1982. 
[17] D. M. J. Tax, D. de Ridder, and R.P.W. Duin, "Support vector classifiers: A first look," in: H.E. Bal, H. Corporaal, P.P. Jonker, J.F.M. Tonino (eds.), Proceedings of 3rd Annual Conference of the Advanced School for Computing and Imaging (Heijen, NL, June 2-4), ASCI, Delft, pp. 253-258, 1997.

[18] J. A. Gualtieri, "The support vector machine (SVM) algorithm for supervised classification of hyperspectral remote sensing data," In G. Camps-Valls and L. Bruzzone (eds) Kernel Methods for Remote Sensing Data Analysis, Wiley, Chichester, in press, 2009.

[19] M. Pal, and P. M. Mather, "Assessment of the effectiveness of support vector machines for hyperspectral data," Future Generation Computer Systems, vol. 20, no. 7, pp. 1215-1225, October 2004.

[20] M. Pal and P. M. Mather, "Some issue in classification of DAIS hyperspectral data," International Journal of Remote Sensing, vol. 27, no. 14, pp. 2895-2916, July 2006.

[21] F. Melgani and L. Bruzzone, "Classification of hyperspectral remote sensing images with support vector machines," IEEE Transaction of Geoscience and Remote Sensing, vol. 42, no. 8, pp. 1778-1790, August 2004.

[22] I. Guyon, J. Weston, S. Barnhill, and V. N. Vapnik, "Gene selection for cancer classification using support vector machines," Machine Learning, vol. 46, no. 1-3, pp. 389-422, Jan. 2002.

[23] A. Gidudu and H. Ruther, "Comparison of feature selection techniques for SVM classification," In 10th Intl. Symposium on Physical Measurements and Spectral Signatures in Remote Sensing (eds M.E. Schaepman, S. Liang, N.E. Groot, and M. Kneubühler), Intl. Archives of the Photogrammetry, Remote Sensing and Spatial 
Information Sciences, Vol. XXXVI, Part 7/C50, p. 258-263, 2007. ISPRS, Davos (CH). ISSN 1682-1777.

[24] H. Liu, "Evolving feature selection," IEEE Intelligent Systems, vol. 20, pp. 64-76, November 2005.

[25] H. Liu and H. Motoda, Feature Extraction, Construction and Selection: A Data Mining Perspective. Massachusetts: Kluwer Academic Publishers, 1998.

[26] P. M. Mather, Computer Processing of Remotely-Sensed Images: An Introduction. Third Edition, Chichester: John Wiley and Sons, 2004.

[27] R. Kohavi and G.H. John, "Wrappers for feature subset selection," Artificial Intelligence, vol. 97, no. 1-2, pp. 273-324, March 1997.

[28] I. Guyon and A. Elisseeff, "An introduction to variable and feature selection," Journal of Machine Learning Research, vol. 3, pp. 1157-1182, March 2003.

[29] M. Dash and H. Liu, "Feature selection for classification," Intelligent Data Analysis: An International Journal, vol.1, no. 3, pp.131-156, 1997.

[30] A. Jain and D. Zongker, "Feature selection: evaluation, application, and small sample performance," IEEE Transactions on Pattern Analysis and Machine Intelligence, vol. 19, no. 2, pp. 153-158, February 1997.

[31] T. Kavzoglu and P. M. Mather, "The role of feature selection in artificial neural network applications," International Journal of Remote Sensing, vol. 23, no 15, pp. 2787-2803, Aug. 2002.

[32] S. B. Serpico and L. Bruzzone, "A new search algorithm for feature selection in hyperspectral remote sensing images," IEEE Transactions on Geoscience and Remote Sensing, vol. 39, no. 7, pp. 1360-1367, July 2001. 
[33] M. Pal, "Support vector machine-based feature selection for land cover classification: a case study with DAIS hyperspectral data," International Journal of Remote Sensing, vol. 27, no. 14, pp. 2877-2894, July 2006.

[34] J. Loughrey and P. Cunningham, “Overfitting in wrapper-based feature subset selection: the harder you try the worse it gets," Research and Development in Intelligent Systems XXI (Max Bramer, Frans Coenen and Tony Allen, eds.), Springer, London, pp. 33-43, 2004.

[35] G.H. Halldorsson, J.A. Benediktsson, J.R. Sveinsson, "Source based feature extraction for support vector machines in hyperspectral classification," IEEE Geoscience and Remote Sensing Symposium, vol. 1, pp. 536-539, 20-24 Sept. 2004.

[36] O. Barzilay and V. L. Brailovsky, "On domain knowledge and feature selection using a support vector machine," Pattern recognition Letters, vol. 20, no. 5, pp. 475-484, May 1999.

[37] A. Navot, R. Gilad-Bachrach, Y. Navot, and N. Tishby, "Is feature selection still necessary?" Lecture notes in computer science, Berlin Heidelberg: SpringerVerlag, vol. 3940, pp. 127-138, 2006.

[38] Y. Bengio, O. Delalleau, and N. Le Roux, "The curse of highly variable functions for local kernel machines," in: Advances in Neural Information Processing Systems, MIT Press, vol.18, pp. 107-114, 2006.

[39] D. Francois, V. Wertz, and M. Verleysen, "About the locality of kernels in high dimensional space," International Symposium on Applied Stochastic Models and Data Analysis, Brest, France, 17-20 May, pp. 238-245, 2005. 
[40] B. Scholkopf, S. Mika, C.J.C. Burges, P. Knirsch, K.R. Muller, G. Ratsch, and A.J. Smola, "Input space versus feature space in kernel-based methods," IEEE Transactions on Neural Networks, vol.10, no. 5, pp.1000-1017, September 1999.

[41] S. Geman, E. Bienenstock and R. Doursat, "Neural networks and the bias/variance dilemma," Neural Computation, vol. 4, no. 1, pp. 1-58, Jan. 1992.

[42] J. L. Fleiss, B. Levin, and M. C. Paik, Statistical Methods for Rates \& Proportions. Third edition, New York: Wiley-Interscience, 2003

[43] G. M. Foody, "Classification accuracy comparison: hypothesis tests and the use of confidence intervals in evaluations of difference, equivalence and non-inferiority," Remote Sensing of Environment, vol. 113, pp. 1658-1663, 2009.

[44] B. Boser, I. Guyon, and V. N. Vapnik, "A training algorithm for optimal margin classifiers," Proceedings of $5^{\text {th }}$ Annual Workshop on Computer Learning Theory, Pittsburgh, PA: ACM, pp.144-152, 1992.

[45] N. Cristianini, and J. Shawe-Taylor, An Introduction to Support Vector Machines and other Kernel-based Learning Methods. Cambridge, UK: Cambridge University Press, 2000.

[46] G.M. Foody and A. Mathur, "A relative evaluation of multiclass image classification by support vector machines," IEEE Transaction of Geoscience and Remote Sensing, vol. 42, no. 6, pp. 1335-1343, June 2004.

[47] G. Camps-Valls and L. Bruzzone, Kernel Methods for Remote Sensing Data Analysis (eds), Wiley, Chichester, in press.

[48] P. Strobl, R. Richter, F. Lehmann, A. Mueller, B. Zhukov, and D. Oertel, "Preprocessing for the airborne imaging spectrometer DAIS 7915," SPIE Proceedings, vol. 2758, pp. 375-382, 1996. 
[49] Aviris NW Indiana's Indian Pines, 1992, data set [online]. Available Online: ftp://ftp.ecn.purdue.edu/biehl/MultiSpec/92AV3C.lan (original files) and ftp://ftp.ecn.purdue.edu/biehl/PC_MultiSpec/ThyFiles.zip (ground truth).

[50] G.M. Foody and M.K. Arora, "An evaluation of some factors affecting the accuracy of classification by an artificial neural network," International Journal of Remote Sensing, vol. 18, no. 4, pp. 799-810, March 1997.

[51] G. M. Foody, A. Mathur, C. Sanchez-Hernandez, D. S. Boyd, "Training set size requirements for the classification of a specific class," Remote Sensing of Environment, vol. 104, no. 1, pp. 1-14, Sept. 2006.

[52] M. Pal and P.M. Mather, "An assessment of the effectiveness of decision tree methods for land cover classification," Remote Sensing of Environment, vol. 86, no. 4, pp. 554-565, October 2003.

[53] T. G. Van Niel, T. R. McVicar, and B. Datt, "On the relationship between training sample size and data dimensionality of broadband multi-temporal classification," Remote Sensing of Environment, vol. 98, no. 4, pp. 468-480, October 2005.

[54] T.G. Dietterich, "Approximate statistical tests for comparing supervised classification learning algorithms," Neural Computation, vol.10, no. 7, pp. 18951923, October 1998.

[55] G.M. Foody, "Thematic map comparison: evaluating the statistical significance of differences in classification accuracy," Photogrammetric Engineering and Remote Sensing, vol.70, no. 5, pp.627-633, May 2004.

[56] D. G. Altman and J. M. Bland, "Absence of evidence is not evidence of absence," British Medical Journal, vol. 311, pp. 485, Aug. 1995. 
[57] M. A. Hall and L. A. Smith, "Feature subset selection: a correlation based filter approach," International Conference on Neural Information Processing and Intelligent Information Systems, Springer, pp. 855-858, 1997.

[58] W.H. Press, Numerical Recipes in C. Cambridge: University Press, 1988.

[59] H. Peng, F. Long and C. Ding, "Feature selection based on mutual information: criteria of max-dependency, max-relevance, and min-redundancy," IEEE Transactions on Pattern Analysis and Machine Intelligence, vol. 27, no. 8, pp. 1226-1238, August 2005.

[60] L. Breiman, "Random forests," Machine Learning, vol. 45, no. 1, pp. 5-32, October 2001.

[61] L. Breiman, "Bagging predictors,” Machine Learning, vol. 24, no. 2, pp. 123-140, August 1996.

[62] R. Díaz-Uriarte and S.A. de Andrés, "Gene selection and classification of microarray data using random forest," BMC Bioinformatics, 7:3, 2006.

[63] C.-W. Hsu, and C.-J. Lin, “A comparison of methods for multi-class support vector machines," IEEE Transactions on Neural Networks, vol. 13, no. 2, pp. 415-425, March 2002.

[64] M. Pal, "Multiclass approaches for support vector machine based land cover classification," 8th Annual International conference, Map India, http://www.mapindia.org/2005/papers/pdf/54.pdf (accessed on 12/11/2008), 2005.

[65] S. Knerr, L. Personnaz and G. Dreyfus, "Single-layer learning revisited: A stepwise procedure for building and training neural network," Neurocomputing: Algorithms, Architectures and Applications, NATO ASI, Berlin: Springer-Verlag, 1990. 
[66] M. Pal, "Margin based feature selection for hyperspectral data," International Journal of Applied Earth Observations and Geoinformation, vol. 11, no. 3, pp. 212-220, June 2009.

[67] T. M. Cover, "The best two independent measurements are not the two best," IEEE Transactions on Systems, Man, and Cybernetics, vol. SMC-4, pp.116-117, January 1974. 


\section{TABLE CAPTIONS}

Table I. Difference between peak accuracy and that derived from the use of all 65 features of DAIS dataset for the results summarised in Fig. 1. The $Z$ value stated was derived from the McNemar test. For the one-sided test adopted a difference is significant at the 0.05 level if $Z>1.64$.

Table II. Difference between peak accuracy and that derived from the use of all 185 features of AVIRIS dataset for the results summarised in Fig. 2. The $Z$ value stated was derived from the McNemar test. For the one-sided test adopted a difference is significant at the 0.05 level if $Z>1.64$.

Table III. Results of the application of the 4 feature selection methods using DAIS dataset highlighting characteristics of the classification based on each training set size that was of most comparable accuracy to that derived without feature selection.

Table IV. Selected features with different data sets and the number of common features selected by various approaches using DAIS dataset.

Table V. Summary of the test for the difference in accuracy between the peak accuracy and that derived from the use of the full feature set using DAIS dataset. Values in bracket gives the number of features providing peak classification accuracy, shown in Fig. 2-4. The $Z$ value stated was derived from the McNemar test. For the one-sided test adopted a difference is significant at the 0.05 level if $Z>1.64$

Table VI. Difference and non-inferiority test results based on $95 \%$ confidence interval on the estimated difference in accuracy from the peak value for feature sets selected with the SVM-RFE using DAIS dataset; based on training set of 100 cases per-class with peak accuracy of $93.13 \%$ with 35 features.

Table VII. Difference and non-inferiority test results based on 95\% confidence interval on the estimated difference in accuracy from the peak value for feature sets selected with the random forest using DAIS dataset; based on training set of 100 cases per-class with peak accuracy of $92.34 \%$ with 35 features.

Table VIII. Difference and non-inferiority test results based on $95 \%$ confidence interval on the estimated difference in accuracy from the peak value for feature sets selected with the mRMR using DAIS dataset; based on training set of 100 cases per-class with peak accuracy of $92.45 \%$ with 45 features. 
Table I.

Training set size per class

\begin{tabular}{|c|c|c|c|c|c|c|}
\hline & 8 pixels & 15 pixels & 25 pixels & 50 pixels & 75 pixels & 100 pixels \\
\hline $\begin{array}{l}\text { Peak accuracy, \% } \\
\text { (number of } \\
\text { features) }\end{array}$ & $74.79(35)$ & $81.21(35)$ & $84.45(35)$ & $88.47(40)$ & $91.13(50)$ & $92.53(50)$ \\
\hline $\begin{array}{l}\text { Accuracy with } 65 \\
\text { features }(\%)\end{array}$ & 69.79 & 77.05 & 81.66 & 87.58 & 90.63 & 91.76 \\
\hline Difference $(\%)$ & 5.00 & 4.16 & 2.79 & 0.89 & 0.50 & 0.77 \\
\hline $\mathrm{Z}$ value & 6.04 & 5.35 & 4.02 & 1.69 & 1.48 & 2.22 \\
\hline
\end{tabular}


Table II.

Training set size per class

8 pixels $\quad 15$ pixels $\quad 25$ pixels $\quad 50$ pixels $\quad 75$ pixels $\quad 100$ pixels

Peak accuracy, \% (number of $67.53(95) \quad 69.49(95) \quad 74.21(130) \quad 81.94(130) \quad 83.65(120) \quad 85.21(125)$ features)

Accuracy with 185 features $(\%)$

Difference (\%)

8.36

5.01

4.02

4.19

4.76

3.75

$\mathrm{Z}$ value

9.44

5.92

8.77

6.92

7.18

6.10

8.77

6.92

.


Table III.

\begin{tabular}{|c|c|c|c|c|c|c|c|c|c|c|c|c|}
\hline \multirow{3}{*}{$\begin{array}{l}\text { Feature } \\
\text { selection } \\
\text { Method }\end{array}$} & \multicolumn{12}{|c|}{ Training set size per class } \\
\hline & \multicolumn{2}{|c|}{8 pixels } & \multicolumn{2}{|c|}{15 pixels } & \multicolumn{2}{|c|}{25 pixels } & \multicolumn{2}{|c|}{50 pixels } & \multicolumn{2}{|c|}{75 pixels } & \multicolumn{2}{|c|}{100 pixels } \\
\hline & $\begin{array}{l}\text { Accuracy } \\
(\%)\end{array}$ & $\begin{array}{l}\text { Feature } \\
\text { size }\end{array}$ & $\begin{array}{l}\text { Accuracy } \\
(\%)\end{array}$ & $\begin{array}{l}\text { Feature } \\
\text { size }\end{array}$ & $\begin{array}{l}\text { Accuracy } \\
(\%)\end{array}$ & $\begin{array}{l}\text { Feature } \\
\text { size }\end{array}$ & $\begin{array}{l}\text { Accuracy } \\
(\%)\end{array}$ & $\begin{array}{l}\text { Feature } \\
\text { size }\end{array}$ & $\begin{array}{l}\text { Accuracy } \\
(\%)\end{array}$ & $\begin{array}{l}\text { Feature } \\
\text { size }\end{array}$ & $\begin{array}{l}\text { Accuracy } \\
(\%)\end{array}$ & $\begin{array}{l}\text { Feature } \\
\text { size }\end{array}$ \\
\hline None & 69.29 & 65 & 74.82 & 65 & 80.58 & 65 & 87.10 & 65 & 90.71 & 65 & 91.76 & 65 \\
\hline $\begin{array}{l}\text { SVM- } \\
\text { RFE }\end{array}$ & 69.84 & 4 & 75.39 & 10 & 81.68 & 7 & 87.45 & 15 & 90.87 & 16 & 91.89 & 13 \\
\hline mRMR & 69.71 & 8 & 76.34 & 11 & 81.02 & 12 & 87.13 & 13 & 90.87 & 42 & 91.84 & 37 \\
\hline CFS & 69.50 & 4 & 75.82 & 7 & 82.18 & 8 & 87.11 & 12 & 91.32 & 14 & 91.84 & 17 \\
\hline $\begin{array}{l}\text { Random } \\
\text { forest }\end{array}$ & 71.94 & 6 & 76.39 & 9 & 81.95 & 9 & 87.11 & 14 & 90.82 & 25 & 92.08 & 21 \\
\hline
\end{tabular}


Table IV.

\begin{tabular}{|c|c|c|c|c|c|c|c|}
\hline \multirow{2}{*}{$\begin{array}{l}\text { Feature } \\
\text { selection } \\
\text { approach }\end{array}$} & \multicolumn{6}{|c|}{ Training set size per class } & \multirow{2}{*}{$\begin{array}{l}\text { Number } \\
\text { of } \\
\text { common } \\
\text { features }\end{array}$} \\
\hline & 8 pixels & 15 pixels & 25 pixel & 50 pixel & 75 pixels & 100 pixels & \\
\hline $\begin{array}{l}\text { SVM- } \\
\text { RFE }\end{array}$ & $1,4,35,53$ & $\begin{array}{l}1,4,6,27,32, \\
36,37,50,51, \\
57\end{array}$ & $\begin{array}{l}1,3,4,26,32, \\
37,42\end{array}$ & $\begin{array}{l}1,2,3,4,18, \\
26,27,31,32, \\
36,37,46,48, \\
52,56\end{array}$ & $\begin{array}{l}1,2,3,4,5,26, \\
27,30,31,32, \\
34,36,37,40, \\
52,56\end{array}$ & $\begin{array}{l}1,2,3,21,26, \\
27,30,34,36, \\
37,51,52,56\end{array}$ & 1 \\
\hline mRMR & $\begin{array}{l}10,15,16,17 \\
24,25,49,56\end{array}$ & $\begin{array}{l}9,16,22,24, \\
25,26,32,48, \\
49,50,65\end{array}$ & $\begin{array}{l}9,15,22,24 \\
25,26,29,31 \\
32,48,49,51\end{array}$ & $\begin{array}{l}8,21,22,23, \\
24,25,26,27, \\
28,30,49,50, \\
65\end{array}$ & $\begin{array}{l}2,3,6,7,8,9, \\
10,12,13,14, \\
15,16,17,18, \\
19,20,21,22, \\
23,24,25,26, \\
27,28,29,30, \\
31,32,36,37, \\
38,41,47,48, \\
49,50,51,52, \\
53,63,64,65\end{array}$ & $\begin{array}{l}6,7,8,9,12, \\
13,14,15,16, \\
17,18,19,20, \\
21,22,23,24, \\
25,26,27,28, \\
29,30,31,32, \\
33,38,41,47, \\
48,49,50,51, \\
52,53,63,65\end{array}$ & 3 \\
\hline CFS & $2,10,15,17$ & $\begin{array}{l}3,10,15,23, \\
24,29,36\end{array}$ & $\begin{array}{l}2,5,10,13, \\
21,24,25, \\
29\end{array}$ & $\begin{array}{l}1,2,5,10,21 \\
22,24,25,27 \\
28,30,31\end{array}$ & $\begin{array}{l}1,2,5,9,20, \\
22,27,28,29, \\
31,32,37,40, \\
44\end{array}$ & $\begin{array}{l}1,2,4,13,17 \\
20,24,25,27 \\
28,30,31,32, \\
36,37,39,45\end{array}$ & 0 \\
\hline $\begin{array}{l}\text { Random } \\
\text { forest }\end{array}$ & $\begin{array}{l}14,28,29 \\
30,41,58\end{array}$ & $\begin{array}{l}10,21,22,24, \\
27,30,32,40, \\
41\end{array}$ & $\begin{array}{l}1,2,5,12,21 \\
28,29,31,32\end{array}$ & $\begin{array}{l}1,2,3,4,5,24, \\
25,26,30,31, \\
32,39,42, \\
50\end{array}$ & $\begin{array}{l}1,2,4,5,6,7, \\
23,24,26,27, \\
29,30,31,32, \\
39,41,42,44, \\
49,50,53,61, \\
63,64,65\end{array}$ & $\begin{array}{l}1,2,3,5,22, \\
23,26,27,28, \\
29,30,31,32, \\
39,40,41,42, \\
50,59,63,64\end{array}$ & 0 \\
\hline
\end{tabular}


Table V.

\begin{tabular}{|c|c|c|c|c|c|c|}
\hline \multirow{2}{*}{$\begin{array}{l}\text { Feature } \\
\text { selection } \\
\text { method }\end{array}$} & \multicolumn{6}{|c|}{$Z$ value } \\
\hline & 8 pixel & 15 pixel & 25 pixel & 50 pixel & 75 pixel & 100 pixel \\
\hline SVM-RFE & $11.54(25)$ & $5.19(20)$ & $7.10(15)$ & $2.33(25)$ & $2.35(25)$ & $4.84(35)$ \\
\hline $\begin{array}{l}\text { Random } \\
\text { forest }\end{array}$ & $7.29(10)$ & $5.54(30)$ & $7.84(25)$ & $1.64(20)$ & $0.25(25)$ & $1.67(35)$ \\
\hline mRMR & $8.73(35)$ & $4.80(15)$ & $7.12(20)$ & $4.01(20)$ & $2.65(50)$ & $2.44(45)$ \\
\hline
\end{tabular}


Table VI.

\begin{tabular}{ccccc}
\hline $\begin{array}{c}\text { Number of } \\
\text { features }\end{array}$ & Accuracy $(\%)$ & $\begin{array}{c}\text { Difference from } \\
\text { peak accuracy } \\
(\%)\end{array}$ & $\begin{array}{c}95 \% \text { confidence } \\
\text { interval }\end{array}$ & $\begin{array}{c}\text { Conclusion (at } \\
0.05 \text { level of } \\
\text { significance) }\end{array}$ \\
\hline 5 & 81.82 & 11.31 & $11.298-11.322$ & Different \\
10 & 90.40 & 2.73 & $2.721-2.739$ & Different \\
15 & 92.47 & 0.66 & $0.653-0.667$ & Non-inferior \\
20 & 93.08 & 0.05 & $0.044-0.056$ & Non-inferior \\
25 & 92.74 & 0.39 & $0.384-0.396$ & Non-inferior \\
30 & 93.03 & 0.10 & $0.096-0.104$ & Non-inferior \\
35 & 93.13 & 0.00 & $0.000-0.000$ & ( No change) \\
40 & 92.74 & 0.39 & $0.386-0.394$ & Non-inferior \\
45 & 92.37 & 0.76 & $0.755-0.765$ & Non-inferior \\
50 & 91.97 & 1.16 & $1.154-1.166$ & Different \\
55 & 91.92 & 1.21 & $1.204-1.216$ & Different \\
60 & 91.95 & 1.18 & $1.174-1.186$ & Different \\
65 & 91.76 & 1.37 & $1.364-1.376$ & Different \\
\hline
\end{tabular}


Table VII.

\begin{tabular}{ccccc}
\hline $\begin{array}{c}\text { Number of } \\
\text { features }\end{array}$ & Accuracy $(\%)$ & $\begin{array}{c}\text { Difference from } \\
\text { peak accuracy } \\
(\%)\end{array}$ & $\begin{array}{c}95 \% \text { confidence } \\
\text { interval }\end{array}$ & $\begin{array}{c}\text { Conclusion (at } \\
\text { 0.05 level of } \\
\text { significance) }\end{array}$ \\
\hline 5 & 79.37 & 12.97 & $12.958-12.982$ & Different \\
10 & 89.58 & 2.76 & $2.751-2.769$ & Different \\
15 & 90.47 & 1.87 & $1.862-1.878$ & Different \\
20 & 91.61 & 0.73 & $0.724-0.736$ & Non-inferior \\
25 & 91.76 & 0.58 & $0.573-0.587$ & Non-inferior \\
30 & 91.50 & 0.84 & $0.835-0.845$ & Non-inferior \\
35 & 92.34 & 0.00 & $0.000-0.000$ & (No change) \\
40 & 92.29 & 0.05 & $0.046-0.054$ & Non-inferior \\
45 & 92.13 & 0.21 & $0.205-0.215$ & Non-inferior \\
50 & 91.92 & 0.42 & $0.414-0.426$ & Non-inferior \\
55 & 91.89 & 0.45 & $0.444-0.456$ & Non-inferior \\
60 & 91.71 & 0.63 & $0.623-0.637$ & Non-inferior \\
65 & 91.76 & 0.58 & $0.573-0.587$ & Non-inferior \\
\hline
\end{tabular}


Table VIII.

\begin{tabular}{ccccc}
\hline $\begin{array}{c}\text { Number of } \\
\text { features }\end{array}$ & Accuracy $(\%)$ & $\begin{array}{c}\text { Difference from } \\
\text { peak accuracy } \\
(\%)\end{array}$ & $\begin{array}{c}95 \% \text { confidence } \\
\text { interval }\end{array}$ & $\begin{array}{c}\text { Conclusion (at } \\
0.05 \text { level of } \\
\text { significance) }\end{array}$ \\
\hline 5 & 80.97 & 11.48 & $11.468-11.492$ & Different \\
10 & 88.5 & 3.95 & $3.940-3.960$ & Different \\
15 & 88.82 & 3.63 & $3.620-3.640$ & Different \\
20 & 91.24 & 1.21 & $1.202-1.218$ & Different \\
25 & 91.58 & 0.87 & $0.862-0.878$ & Non-inferior \\
30 & 91.03 & 1.42 & $1.413-1.427$ & Different \\
35 & 91.53 & 0.92 & $0.914-0.926$ & Non-inferior \\
40 & 92.16 & 0.29 & $0.286-0.294$ & Non-inferior \\
45 & 92.45 & 0.00 & $0.000-0.000$ & (No change) \\
50 & 92.34 & 0.11 & $0.106-0.114$ & Non-inferior \\
55 & 92.24 & 0.21 & $0.206-0.214$ & Non-inferior \\
60 & 92.11 & 0.34 & $0.335-0.345$ & Non-inferior \\
65 & 91.76 & 0.69 & $0.685-0.696$ & Non-inferior \\
\hline
\end{tabular}




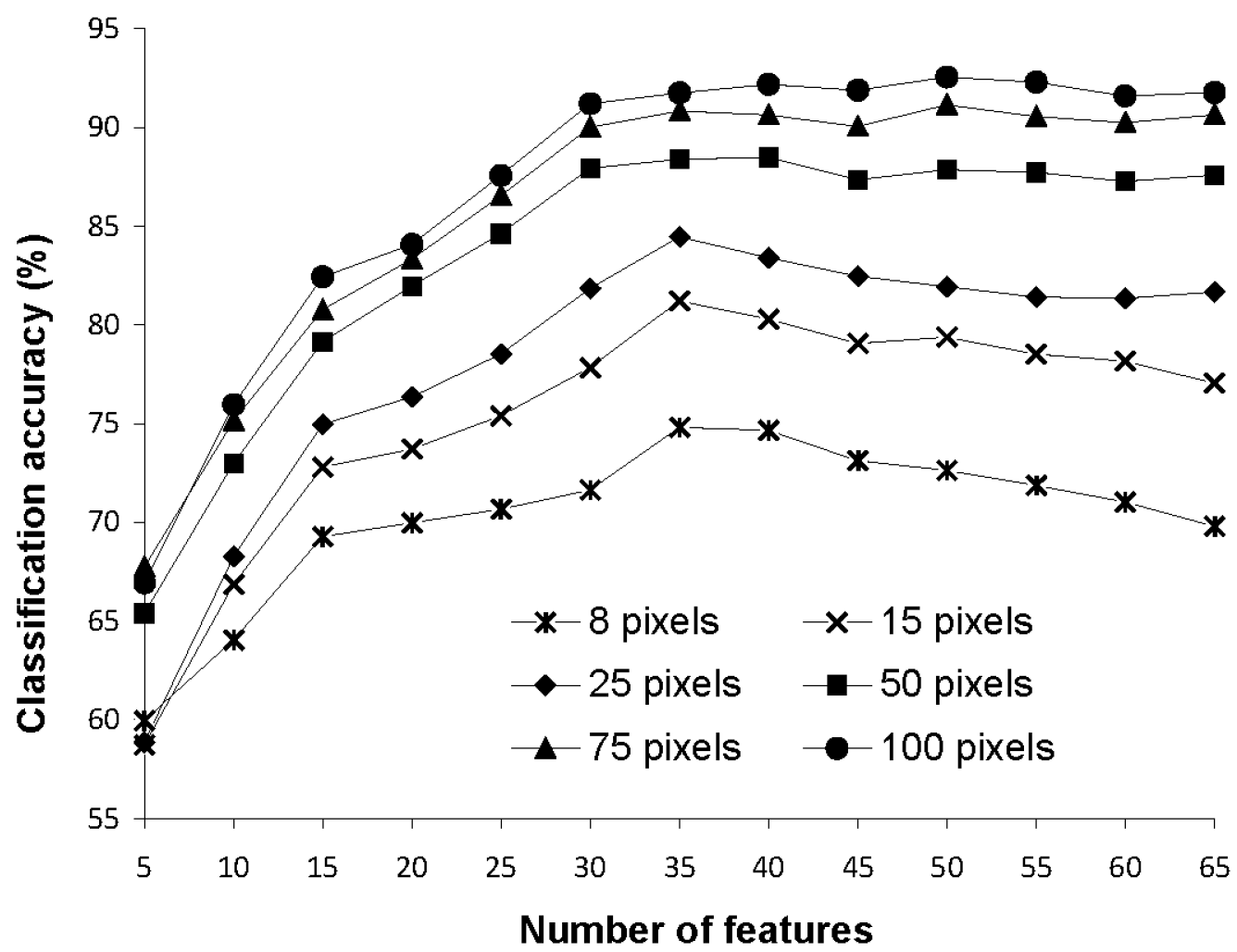

Fig. 1. Variation of classification accuracy with number of features for analyses based on training sets of differing size using DAIS dataset. 


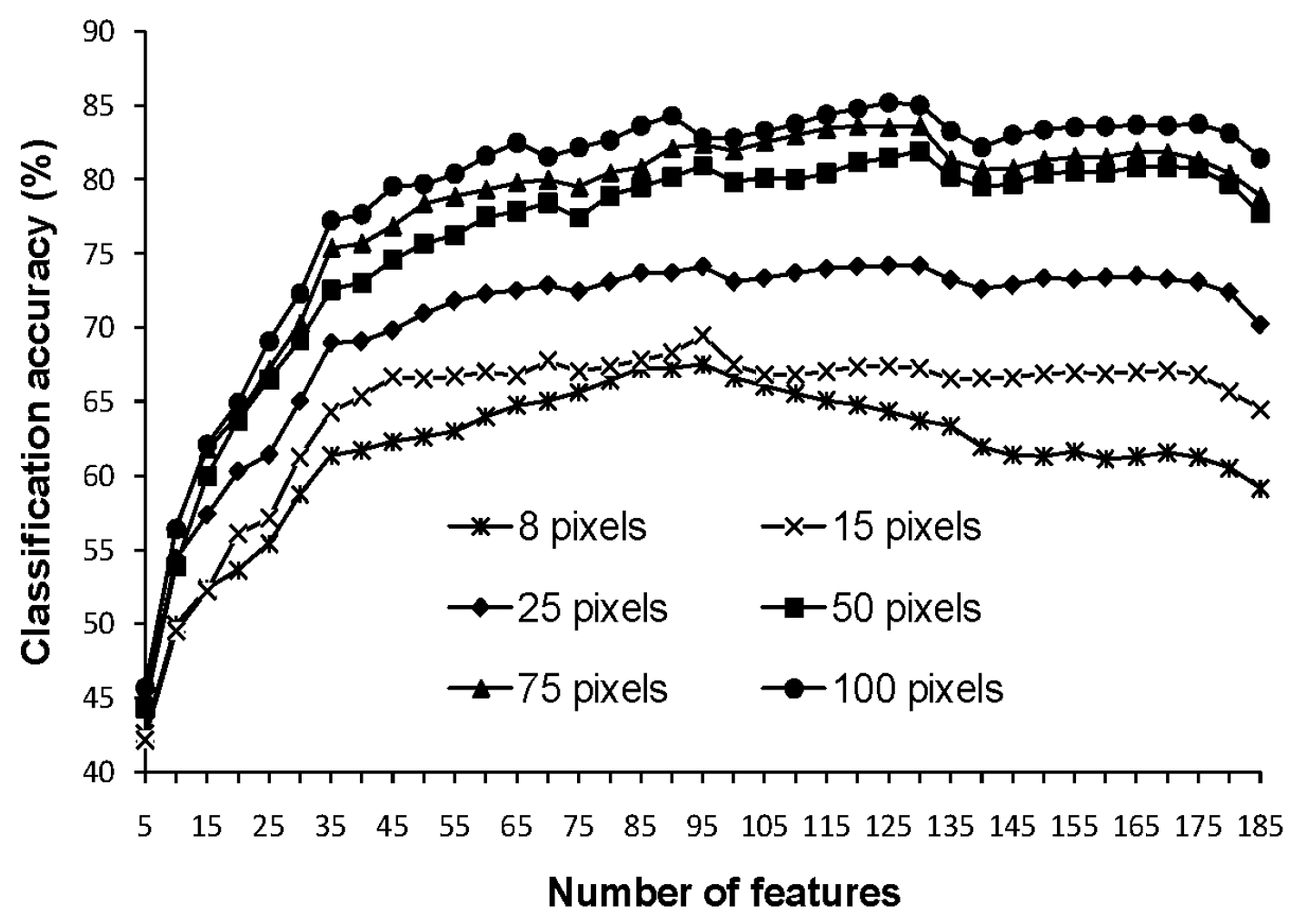

Fig. 2. Variation of classification accuracy with number of features for analyses based on training sets of differing size using AVIRIS dataset. 


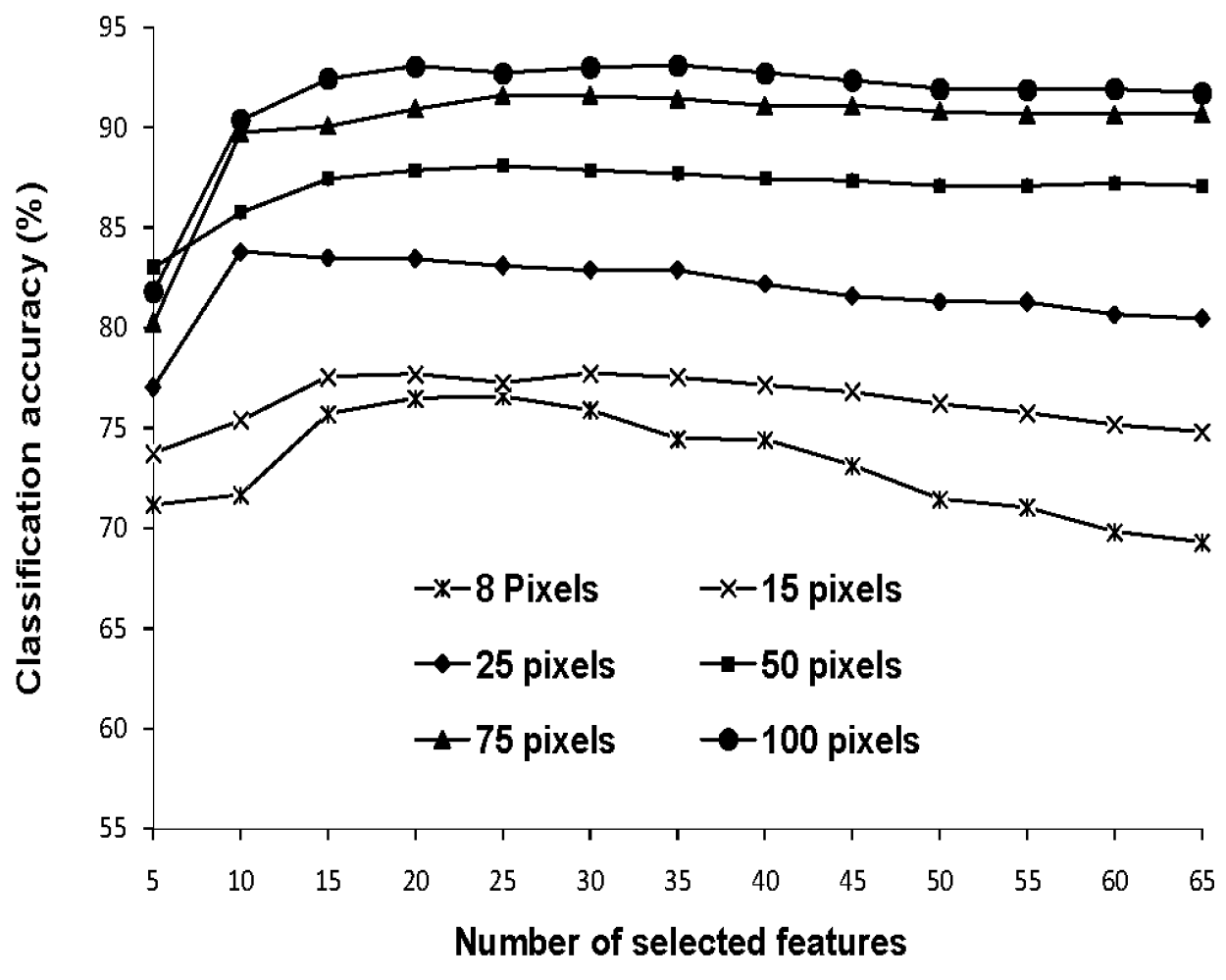

Fig. 3. Relationship between classification accuracy and the number of features selected by the SVM-RFE using DAIS dataset. 


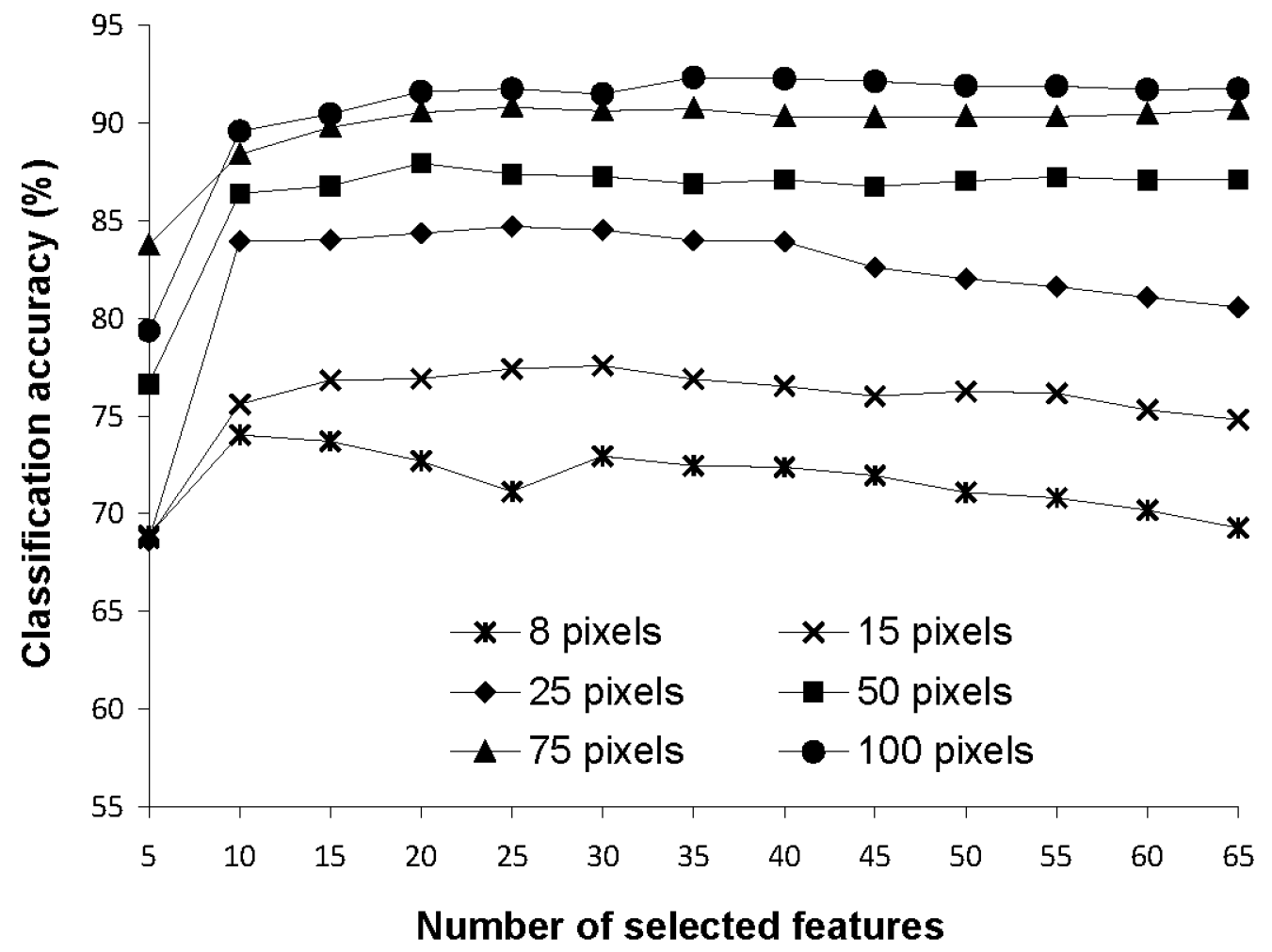

Fig. 4. Relationship between classification accuracy and the number of features selected by the random forest using DAIS dataset. 


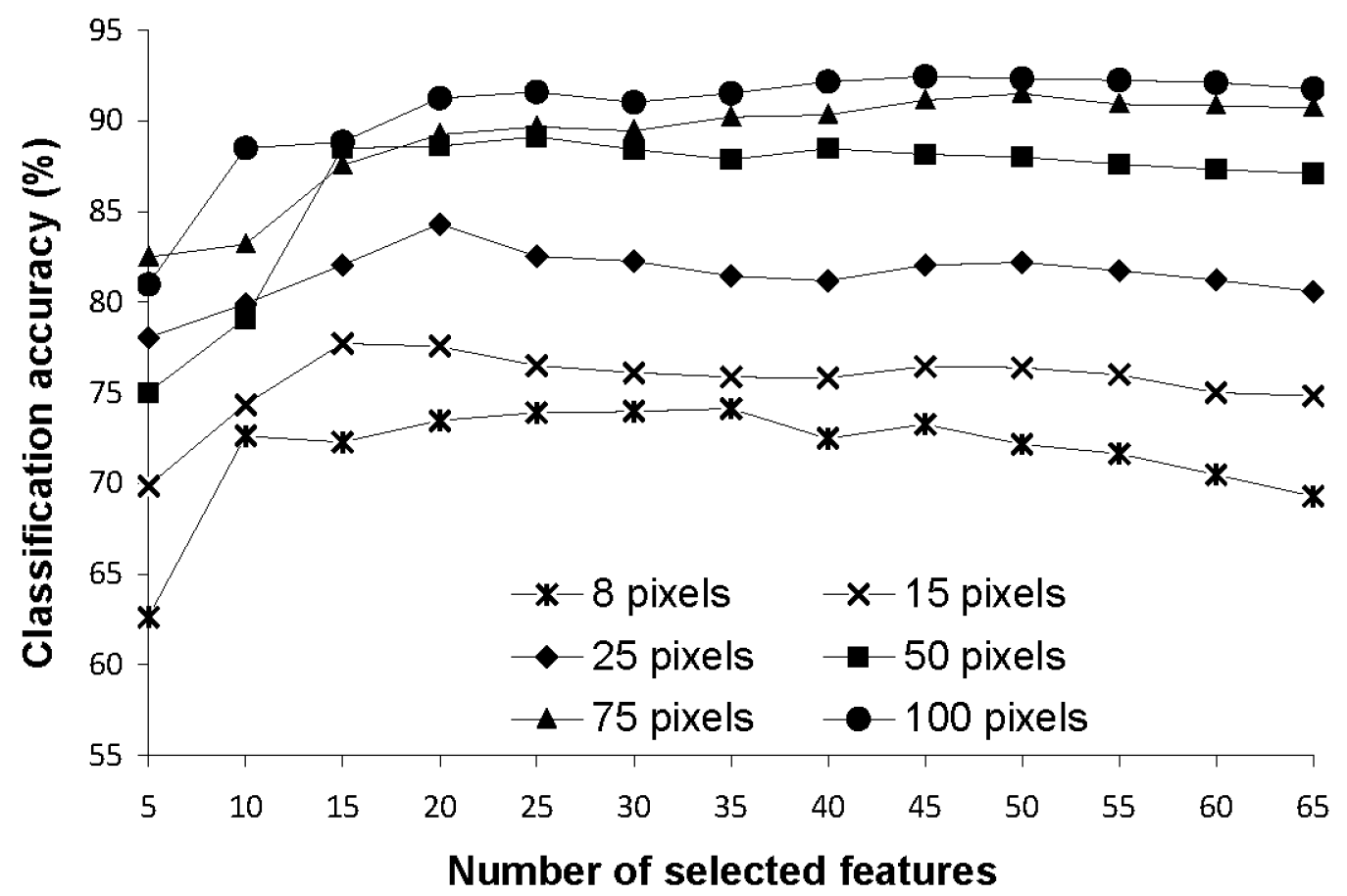

Fig. 5. Relationship between classification accuracy and the number of features selected by the mRMR using DAIS dataset. 